\title{
Arqueología urbana en Ceuta (2000-2005)
}

Fernando Villada Paredes

Arqueólogo Municipal. Ciudad Autónoma de Ceuta

\section{RESUMEN}

Se exponen los avances en el conocimiento de los yacimientos arqueológicos más significativos de Ceuta en los últimos cinco años. Ha sido una etapa donde se ha dado un gran salto en la investigación. Se valoran los importantes resultados obtenidos a partir de la Carta Arqueológica de Ceuta como marco para la gestión y planificación del Patrimonio de la ciudad. Se exponen los avances en el conocimiento de los últimos años producidos en la Arqueología de Ceuta. Se analizan sucintamente los resultados de algunos: Benzú, yacimiento fenicio de la Plaza de la Catedral, Septem Fratres, basílica tardorromana, hallazgos medievales. Se expone la necesidad de control y seguimiento arqueológicos en una ciudad en continuo crecimiento. Se expone una defensa de la educación, con la fomentación de multitud de actos ante los escolares de la ciudad en la línea de conocimiento y defensa del patrimonio arqueológico e histórico de Ceuta.

\section{RÉSUMÉ · L'archéologie urbaine à Ceuta (2000-2005)}

On explique les progrès dans la connaissance des gisements archéologiques les plus significatifs de Ceuta durant les cinq dernières années. Ce fut une étape ou il y eut un grand saut dans la recherche. On met en valeur les résultats importants obtenus à partie de la Carte Archéologique de Ceuta comme cadre pour la gestion et la planification du Patrimoine de la ville. On expose les progrès dans la connaissance des dernières années produites dans l'archéologie de Ceuta. On analyse succinctement les résultats de certains: Benzú gisement phénicien de la Place de la Cathédrale, Septem Fratres, une basilique postromaine, découvertes médiévales. On expose la nécessité de contrôle et de suivi archéologiques dans une ville en perpétuel développement. On expose une défense de l'éducation, en favorisant de multiples actes devant les élèves de la ville dans la ligne de connaissance et de défense du patrimoine archéologique et historique de Ceuta.

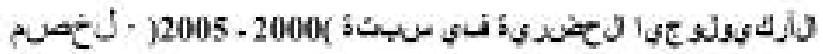

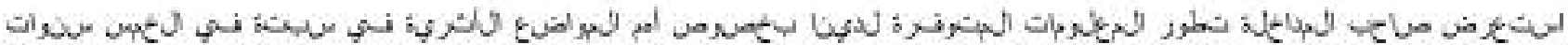

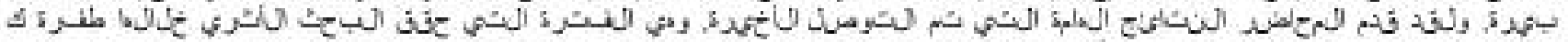

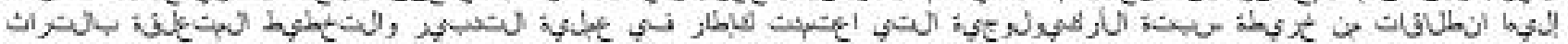

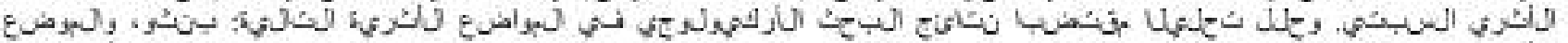

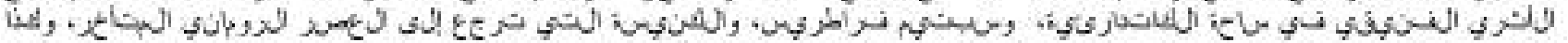

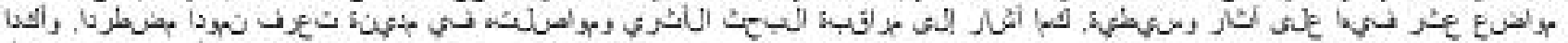

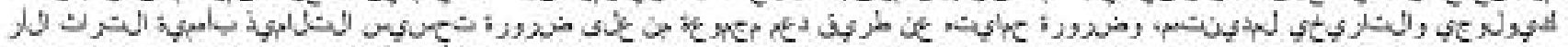
Sill
} 
Podría decirte de cuantos peldaños son sus calles en escalera, de qué tipo los arcos de sus soportales, qué chapas de zinc cubren los techos; pero sé ya que sería como no decirte nada. No está hecha de ésto la ciudad, sino de relaciones entre las medidas de su espacio y los acontecimientos de su pasado [...]

Pero la ciudad no dice su pasado, lo contiene como las líneas de una mano, escrito en los ángulos de las calles, en las rejas de las ventanas, en los pasamanos de las escaleras, en las antenas de los pararrayos, en las astas de las banderas, surcado a su vez cada segmento por raspaduras, muescas, incisiones, cañonazos.

Italo Calvino, Las ciudades invisibles

Nuestro conocimiento de la historia de la ciudad de Ceuta ha avanzado de forma notable en los últimos decenios. Entre otros, el desarrollo experimentado por la investigación arqueológica es uno de los factores que más ha influido en este proceso. Especialmente en los últimos años una política más decidida de protección e investigación del patrimonio arqueológico ceutí, aunque no exenta de las dificultades propias de la arqueología urbana, ha contribuido a aportar nuevos datos que permiten empezar a conocer periodos hasta el momento inéditos en la historia de la Ciudad y profundizar decisivamente en otros momentos mejor conocidos. El objetivo de estas páginas no es otro que presentar un balance de algunas de las intervenciones más destacadas, mostrando en qué medida ha avanzado nuestro conocimiento sobre los distintos periodos a raíz de estas investigaciones.

Dado el espacio disponible hemos decidido circunscribir el ámbito temporal de este trabajo al último lustro. Pensando en aquellos investigadores interesados en profundizar en las diversas cuestiones planteadas hemos incluido una bibliografía complementaria que confiamos supla la necesaria brevedad con que deben abordarse estas cuestiones en esta ocasión. No obstante, al tratarse de hallazgos muy recientes, muchos de ellos permanecen aún inéditos o sólo parcialmente publicados.

Nuestro trabajo se articula en seis apartados. En el primero de ellos realizamos una introducción en la que prestamos una especial atención a esbozar una pequeña síntesis de la investigación arqueológica en Ceuta. Después, dedicamos un capítulo específico a presentar brevemente un proyecto de investigación, la Carta Arqueológica Terrestre de Ceuta, dado que a nuestro juicio marca un cambio en la dinámica de la gestión y la investigación del patrimonio arqueológico ceutí. A continuación, y siguiendo un esquema cronológico presentamos las principales novedades de la arqueología ceutí entre 2000-2005 agrupadas siguiendo un esquema cronológico tradicional: prehistoria, protohistoria, edad antigua y edad media.

\section{INTRODUCCIÓN}

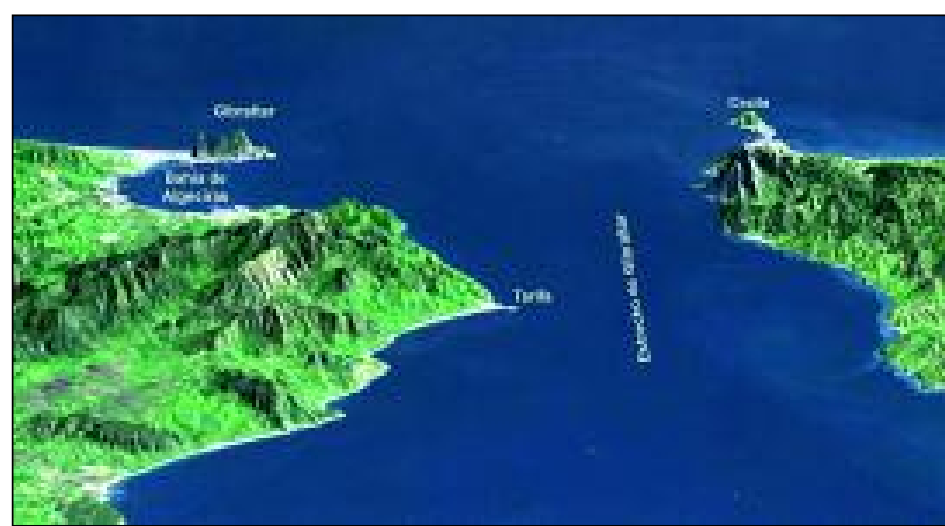

Figura 1. Situación de Ceuta.

La ciudad de Ceuta se encuentra construida sobre una península situada en la embocadura sur del Estrecho de Gibraltar (figura 1).

Su estratégica situación ha generado un devenir histórico rico y variado que tiene su reflejo en un registro arqueológico copioso y de gran interés. Estos "archivos del suelo", como han sido denominados, ofrecen un potencial de conocimiento de indudable valor para la comprensión del fenómeno histórico acaecido en la ciudad.

Los primeros datos que se tienen de una ocupación humana del territorio se remontan a hace unos 270.000 años (Abrigo de Benzú). El actual casco urbano ha estado habitado desde hace unos 2.700 años, lo que ha provocado una modificación más o menos importante del subsuelo y consecuentemente del registro arqueológico. Aunque este proceso de destrucción/construcción y aprovechamiento de estructuras preexistentes, propio de cualquier ciudad histórica, ha estado presente en todo momento, es evidente que el impacto sobre el subsuelo es ahora infinitamente mayor por lo que es necesario adoptar medidas urgentes para paliar en la medida de lo posible esta amenaza. En estas condiciones, la situación del patrimonio arqueológico es muy delicada pues si bien existen muchas oportunidades de realizar intervenciones no siempre se cuenta con los recursos precisos para llevarlas a cabo y transformar los datos obtenidos en conocimiento histórico.

Aunque la mención de hallazgos antiguos y la existencia de monumentos anteriores a la época en que fueron escritos están presentes en numerosos textos y crónicas, el inicio de la investigación arqueológica entendida en un sentido moderno debe fijarse en la década de los 50 del siglo pasado y está estrechamente vinculada a la labor desarrollada por Carlos Posac Mon. Superando grandes obstáculos y la indiferencia cuando no el rechazo de muchos, Posac comenzó sus actividades de salvamento y recuperación de vestigios históricos (Aróstegui, 1998). Su trabajo aportó los primeros datos sobre el periodo romano de Ceuta, confirmando la existencia de una importante factoría de salazones de pescado. Además, su interés no se centró únicamente en la arqueología clásica como era habitual en esos momentos, sino que atendió también a otros periodos más recientes publicando algunos de los primeros artículos sobre arqueología medieval islámica en España (Posac, 1960). Su trabajo "Estudio arqueológico de Ceuta" (Posac, 1962) constituye la primera síntesis histórica de la ciudad en la que los datos procedentes de la investigación arqueológica tienen una relevancia destacada. Él fue también el impulsor de la Sala Municipal de Arqueología de Ceuta, precedente del actual Museo de Ceuta. 
En estos años, se inicia también de la mano de Juan Bravo Pérez una importante labor de recuperación de restos submarinos en el litoral ceutí que alcanzan notable repercusión nacional e internacional (Bravo, 1968, Bravo y Muñoz, 1965; Bernal, ed., 2004).

La década de los 80 e inicios de los 90 viene marcada por la figura de Emilio Fernández Sotelo, director de la Sala Municipal de Arqueología. Su interés se centra en primer lugar en el estudio de las cerámicas medievales, siendo su tesis obra de referencia aún en este campo de estudios (Fernández Sotelo, 1988). Entre sus investigaciones arqueológicas más destacadas deben mencionarse las realizadas en torno a la Basílica Paleocristiana, uno de los monumentos de mayor interés del cristianismo primitivo en la Tingitana (Fernández Sotelo, 1991, 1995 y 2000).

La década de los 90 supuso una ampliación en la nómina de investigadores que trabajan en la ciudad y se caracterizó por la incorporación a la arqueología ceutí de las nuevas técnicas de investigación que habían ido desarrollándose en momentos precedentes en España y Europa. Son de destacar las numerosas aportaciones que a partir de la investigación arqueológica se realizan en este momento sobre la factoría de salazones, especialmente sobre los momentos más tardíos de la misma y en general sobre el periodo romano, la documentación de los primeros niveles de ocupación bizantinos y notables hallazgos de estructuras domésticas del periodo mariní que han dado lugar a numerosas publicaciones (véase bibliografía final).

\section{INSTRUMENTOS DE PROTECCIÓN: CARTAS ARQUEOLÓGICAS TERRESTRE Y SUBMARINA DE CEUTA}

Existe un consenso generalizado entre los gestores del patrimonio histórico en señalar que su identificación es el primer paso para su protección, ya que resulta imposible proteger aquello que no se conoce. Esta afirmación cobra aún mayor relevancia si cabe en el caso del patrimonio arqueológico, una de cuyas características es su "invisibilidad", al permanecer oculto en el subsuelo en su mayor parte. Es también un patrimonio frágil, no renovable y finito que cuando es puesto al descubierto casualmente se encuentra ya en grave riesgo.

Esto ha llevado a desarrollar instrumentos que permitan "proteger lo desconocido, inventariando las sospechas" (Querol y Martínez Díaz, 1996). De esta necesidad se han hecho eco la mayor parte de los documentos internacionales suscritos en relación con la gestión del patrimonio arqueológico. Podemos citar como ejemplo La Carta Internacional para la Gestión del Patrimonio Arqueológico (adoptada por el ICOMOS en 1990) que indica que

"La protección del patrimonio arqueológico debe basarse en el más completo conocimiento posible de su existencia, de su amplitud y de su naturaleza [...]. En consecuencia, el inventario acotado es una obligación fundamental para la protección y gestión"

También el artículo segundo del Convenio Europeo para la protección del patrimonio arqueológico (La Valleta, 1992) señala, entre las políticas de protección,

"the maintenance of an inventory of its archaeological heritage and the designation of protected monuments and areas".

Esta información, incorporada al planeamiento territorial, permite actuaciones preventivas que ayudan a limitar el impacto sobre dicho patrimonio (Rodríguez Termiño, 2003, 203 y ss.).

El primer intento en esa línea en Ceuta es la alegación presentada por J. M. Hita y F. Villada durante el periodo de exposición pública del vigente Plan General de Ordenación Urbana. Teniendo como referencia los datos disponibles entonces se delimitaban una serie de zonas de protección, en las que se proponían actuaciones acordes a las expectativas de afección al patrimonio arqueológico que pudieran producirse en cada caso. La alegación fue aceptada por los redactores del Plan y aceptada por unanimidad por el Pleno del Ayuntamiento. No obstante, no fue incluida en el propio PGOU, sino que su puesta en práctica se pospuso hasta el desarrollo de la normativa de licencias. Tras diversos avatares, fue aprobada en 1996 la Ordenanza Reguladora de la Disciplina Urbanística que contenía un Apéndice de Protección del Patrimonio Arqueológico (BOCCE extraordinario $n^{\circ}$ 9, de 3.09.96). El contenido normativo de este apéndice se articula en cinco apartados que regulan las funciones del Servicio Municipal de Arqueología, cuya creación estaba prevista en la Ordenanza, la tramitación de los expedientes urbanísticos, los niveles, las zonas y las condiciones de protección (Alcalá Velasco, 1998).

Tras su aprobación, la aplicación de lo dispuesto en ella fue muy desigual debido tanto a cierta falta de voluntad de los responsables para exigir su cumplimiento, traducida en la carencia de dotación de medios humanos y financieros para su ejecución, como por el complejo proceso político y administrativo que supuso la adaptación a la nueva realidad autonómica derivada de la entrada en vigor de la Ley Orgánica 1/1995 del Estatuto de Autonomía de la Ciudad de Ceuta y el posterior proceso de traspaso de competencias.

En 1998 la situación era preocupante. El Servicio de Arqueología Municipal no había sido creado y el grado de cumplimiento de esta normativa de protección [...] prácticamente nulo (Alcalá Velasco, 1998, 363). La responsabilidad de la administración era doble. De un lado, no se aplicaban los mecanismos de protección previstos y de otra "la ejecución de las grandes obras públicas y de las promociones inmobiliarias de mayor envergadura, casi siempre corresponde a la Administración (estatal, autonómica y local) o a las sociedades de gestión por ella creadas. Y son precisamente estas operaciones urbanísticas las que inciden en el tema de la salvaguarda del patrimonio arqueológico de forma más patente [aunque] justo es reconocer que algunas ya han comenzado a dar ejemplo" (Alcalá, 1998, 365).

Hoy la situación ha cambiado radicalmente y la aplicación de la ordenanza en los últimos años ha sido más satisfactoria como demuestra el incremento de las intervenciones desarrolladas al amparo de ésta.

En este contexto fue encargado a un equipo dirigido por Bernal Casasola la realización de la Carta Arqueológica Terrestre de Ceuta (CATC). Los trabajos se llevaron a cabo en 2001 y, ante la falta de una reglamentación específica, sus redactores tomaron como marco de referencia la normativa establecida por la Junta de Andalucía.

Concebida como Carta Arqueológica de Riesgo "se ha convertido en un instrumento eficaz para la Ciudad Autónoma en materia de protección y difusión del Patrimonio Histórico" (Bernal Casasola, 2004, 85).

El documento elaborado se estructura en tres apartados. En el primero se llevó a cabo una recopilación y sistematización de la documentación previa (recopilación bibliográfica y hemerográfica, estudio de los fondos del Museo de Ceuta y de 
la documentación administrativa existente). En una segunda fase se realizó una prospección de superficie del término municipal para a continuación proceder a la redacción de la Carta de Riesgo y Prevención. En ella se propuso una nueva zonificación y la delimitación de áreas de servidumbre arqueológica. Se completa con una serie de anexos que incluyen la bibliografía fotocopiada, un dossier de prensa con noticias de hallazgos, copias de los informes de excavación y de los permisos y autorizaciones otorgadas.

Estos instrumentos requieren una constante puesta al día para incorporar los avances de la investigación. La CATC ha sido revisada en 2005, incorporando la información generada en el periodo 2000-2005. Asimismo, ha sido digitalizada para facilitar su uso.

Como señala Bernal, el avance en el conocimiento del patrimonio arqueológico ceutí se ha incrementado tanto cuantitativa como cualitativamente, en porcentajes insospechados, pasando de 11 "yacimientos" identificados a 72 y de 6 estructuras emergentes a 74, la mayoría en el Campo Exterior. Asimismo, se documentaron yacimientos de fases inéditas hasta el momento en la historia de Ceuta como es el caso de los referidos a época prehistórica (Bernal Casasola, 2004).

A pesar de la falta de desarrollo de las propuestas de gestión presentes en la Carta Terrestre, de las dificultades de la prospección en suelo urbano o en parcelas de uso militar, de la falta de integración con la Carta Arqueológica Submarina (vide infra) y de la ambigüedad de ciertos conceptos empleados como por ejemplo el de "yacimiento" (toda Ceuta es un único yacimiento sensu stricto), la Carta Arqueológica Terrestre de Ceuta se ha convertido en el principal instrumento de gestión de este patrimonio en sus diversas vertientes. Ha supuesto también una valiosa contribución al conocimiento de periodos ausentes de Ceuta hasta ese momento.

Paralelamente se encargó a otro equipo de trabajo la Carta Arqueológica y Bionómica del litoral ceutí. En lo referente al patrimonio arqueológico, consistió en la realización de una serie de prospecciones con sonar que dieron como resultado la elaboración de un mapa de "puntos de interés arqueológico" (PIA). A falta de prospecciones con buceadores que permitirán precisar la naturaleza de dichos PIA, en la que se trabaja en estos momentos, y de la integración de los datos obtenidos con los resultados ofrecidos por la CATC, debe considerarse esta Carta Submarina simplemente un paso inicial, necesario pero insuficiente, para la adecuada protección de nuestro patrimonio arqueológico submarino.

\section{ORÍGENES}

Una de las aportaciones de la realización de la CATC fue, como hemos indicado, la localización de diversos "yacimientos" y hallazgos aislados prehistóricos. Entre ellos destacaba el Abrigo y Cueva de Benzú (figura 2), que ha sido objeto de un proyecto de investigación dirigido por José Ramos Muñoz y Darío Bernal Casasola, aún en curso de realización. La publicación en este mismo volumen de un trabajo sobre este proyecto nos permitirá hacer una presentación muy sucinta del mismo.

Se trata en realidad de dos lugares próximos pero distintos. El abrigo presenta una interesante secuencia del Pleistoceno Medio y Superior, en tanto que la cueva fue frecuentada por sociedades tribales neolíticas. Su potencial y la falta de información sobre ambos periodos motivaron una primera excavación en 2002. Las campañas han ido sucediéndose anualmente en el marco de un Convenio de colaboración suscrito entre la Ciudad

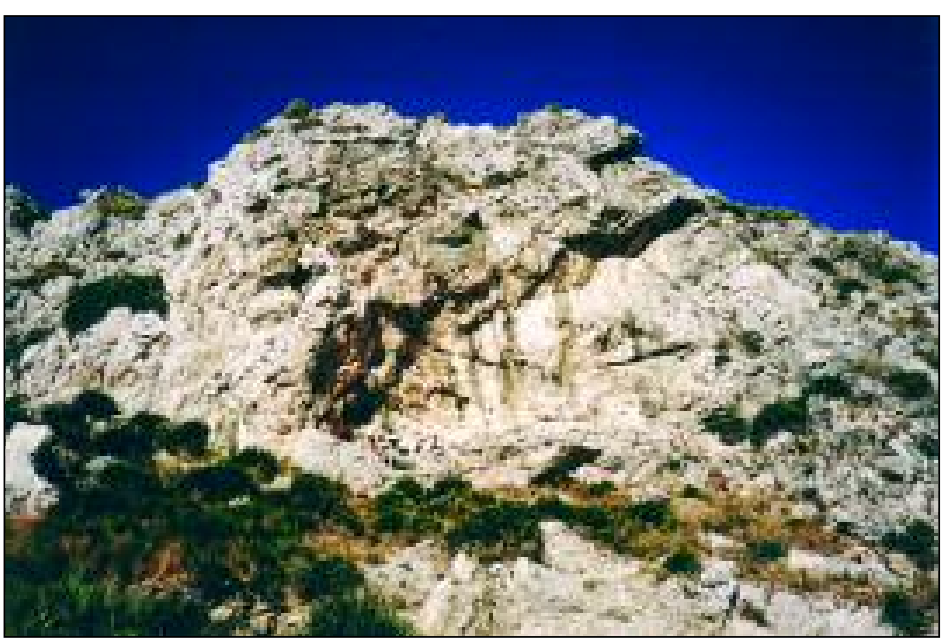

Figura 2. Abrigo y Cueva de Benzú. Autónoma de Ceuta y la Universidad de Cádiz, correspondiendo su financiación a la Ciudad Autónoma, con aportaciones de particulares y asociaciones ceutíes. Hasta el momento, se ha llevado a cabo una completa topografía del yacimiento, la documentación de los artefactos y ecofactos visibles en superficie, análisis medioambientales (palinología, faunísticos, etc.), diversas dataciones absolutas, así como un sondeo de un metro cuadrado de toda la secuencia.

Debe destacarse que en torno al proyecto se ha articulado un eficaz equipo interdisciplinar en el que participan un nutrido grupo de investigadores tanto nacionales como europeos.

Los complejos problemas históricos que plantea el proyecto que se lleva a cabo (contactos prehistóricos entre ambas orillas, evolución antropológica en el extremo occidental norteafricano, la caracterización del proceso de neolitización en la región, etc.) han captado el interés tanto de los ambientes académicos como de los medios de comunicación.

La relevancia de la información obtenida hasta el momento se traduce en el numeroso grupo de publicaciones surgidas, en diversos trabajos presentados en distintos foros nacionales e internacionales y en la constante visita de investigadores y estudiosos. Desde el punto de vista local, ha tenido una importante incidencia en la sociedad ceutí. Diversas conferencias y cursos han sido desarrollados en torno a este proyecto de investigación en Ceuta. Asimismo, los medios de comunicación local recogen con interés el desarrollo del proyecto, propiciando de este modo un acercamiento de los resultados de la investigación al ciudadano.

Consecuencia de éstas y otras actividades de sensibilización ha sido el desarrollo de un programa de voluntariado con resultados muy positivos. Ha permitido la participación de un sector de la población ceutí en la investigación del propio yacimiento, generando una dinámica de trabajo y sensibilización social que se ha extendido a otras actuaciones arqueológicas llevadas a cabo en la Ciudad. El proceso ha culminado en la creación reciente de una Asociación de Amigos del Museo de Ceuta. 


\section{INFLUENCIAS ORIENTALES}

Los datos que se tenían sobre el periodo protohistórico hasta fechas muy recientes se reducían a una serie de hallazgos anfóricos submarinos principalmente recuperados en la bahía de Benzú. Estas ánforas abarcaban un amplio periodo cronológico entre el siglo V a.C. y el siglo II-I a.C. En tierra, habían sido atribuidos incorrectamente a este periodo diversas piezas (Bernal Casasola, 2000; Ramon Torres, 2004).

La excavación arqueológica preventiva (Villada Paredes, Ramon Torres y Suárez Padilla, en prensa) realizada en un solar sito al Oeste de la Catedral de Ceuta en 2004-2005 permitió documentar una compleja y amplia secuencia estratigráfica, caracterizada por presentar evidencias sobre el sustrato geológico, de varios periodos de ocupación: proto-

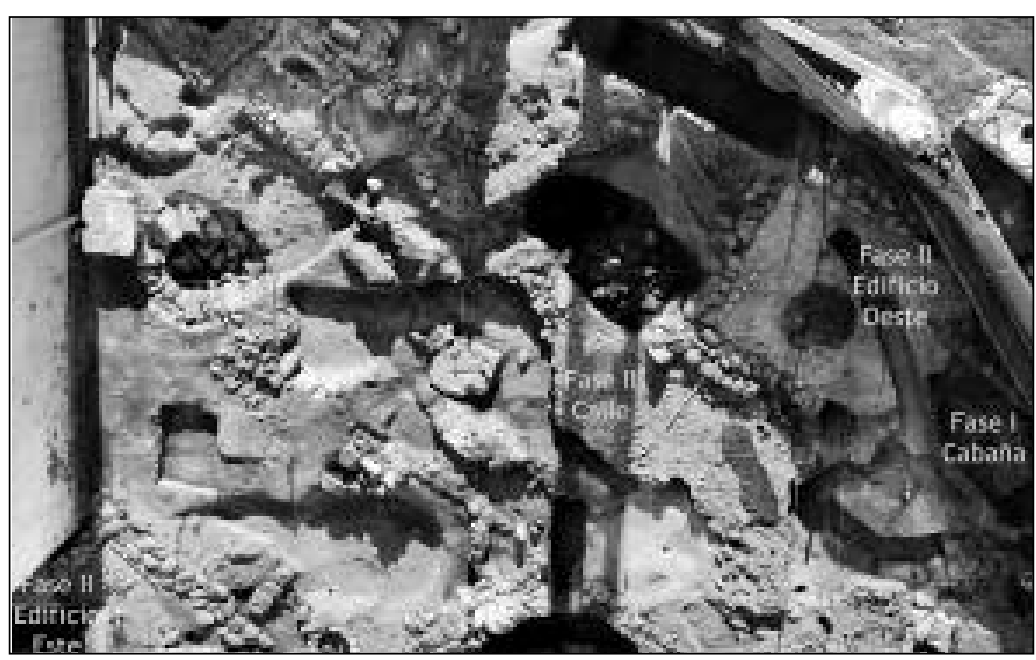

Figura 3. Vista general de la excavación de la Plaza de la Catedral. histórico, romano, medieval, moderno y contemporáneo (figura 3). De todos ellos, sólo del primero se conservan restos constructivos con su secuencia deposicional asociada. Los restantes, que vienen a ocupar en planta más de la mitad del área investigada, se caracterizan por estar asociados exclusivamente a subtrucciones y a fosas con sus correspondientes rellenos.

Como consecuencia de ello, nos encontramos ante un yacimiento con una potencia máxima de un metro, donde los niveles más arcaicos se encuentran prácticamente a la cota de la superficie actual y se han visto sometidos a importantes remociones del subsuelo. Así, resultado de dichas afecciones, se conserva una estratigrafía muy discontinua y en muchos casos inconexa, lo que obliga a realizar un gran esfuerzo de interpretación.

Centrándonos en el periodo protohistórico, presentamos la secuencia de ocupación agrupada en fases constructivas con sus respectivos momentos de uso, abandono y amortización.

\section{Fase I: Primeros indicios del asentamiento (Finales del siglo VIII-primera mitad del VII a.C.)}

A este momento inicial de la secuencia pueden asociarse una serie de estratos de naturaleza deposicional, restos de vertidos domésticos sobre el geológico.

Sólo contamos con retazos de un suelo de cantos rodados directamente apoyado sobre el sustrato geológico, al que se le superpone un estrato con una potencia de unos 20-30 cm, de textura arcillosa, con restos de fauna y material cerámico. En este nivel se han excavado unos hoyos, previsiblemente para ubicar postes. Contorneándolos, se ha conservado en bastante mal estado una estructura interpretada como restos de un pequeño murete de barro endurecido, que pudo definir la planta de una cabaña, con tendencia ligeramente ovalada. Es posible que en un primer momento este lugar fuese ocupado por un hábitat disperso de estructuras perecederas.

\section{Fase II. La urbanización del sector. Mediados del siglo VII a.C.} precedentes

A mediados del siglo VII este espacio se presenta urbanizado, amortizándose los niveles y los restos de construcciones

Los restos de edificios conservados se articulan a partir de un espacio de unos cuatro metros y medio de ancho, orientación N-S, y pavimentado con gravas en su mitad occidental, que ha sido interpretado como una posible calle. Este ámbito será objeto de continuados vertidos de desechos domésticos (circunstancia que contrasta con los espacios intramuros que aparecen prácticamente limpios). En algunos casos se ha documentado incluso la práctica de hogueras realizadas en la propia calle, que serán sepultadas por nuevos aportes de basura.

En el sector que linda con el espacio constructivo situado al E. se localizan una serie de hoyos para postes, paralelos a uno de los edificios. Pueden relacionarse con la existencia de un espacio porticado, abierto hacia la calle.

Dos edificios de planta ortogonal han sido identificados. El primero, al Este, presenta una serie de estructuras de mampostería cuya relación estratigráfica permite plantear la existencia de dos momentos constructivos, que señalan la existencia de transformaciones urbanísticas relativamente frecuentes. A una primera fase corresponden los restos de dos paramentos, perpendiculares entre sí, realizados con cantos rodados de pequeño y medio tamaño. Sobre los restos de este primer edificio, pero manteniendo aproximadamente su orientación, se localiza parte de una vivienda en aceptable estado de conservación, que contaba al menos con cuatro estancias. Sus zócalos son de mampostería, con caras vivas, algo diferente de la usada en el edificio precedente. Los suelos dispuestos al sur aparecen sobre el geológico, y los situados más al norte amortizan las estructuras de la fase precedente. Están realizados con grava de pequeño tamaño, bien compactada, que alterna con suelos de arcilla rojiza con indicios de rubefacción. En algunos casos se observa cómo se repararon los suelos, sustituyendo los de barro por empedrados. La presencia de niveles limpios de micaesquistos depositados sobre los suelos quizás pueda ponerse en relación con su uso en posibles cubiertas planas de los edificios.

Al lado Oeste del tramo de calle investigado se instaló otra vivienda, bastante distinta a la descrita con anterioridad. Se trata de un edificio de planta rectangular, con una única estancia conservada, aunque pudo tener otras hoy perdidas. Los muros, de unos $60 \mathrm{~cm}$. de ancho, están realizados con grandes mampuestos rodados y ligados con barro. El piso interior está sobreelevado respecto a la calle. En la estancia se dispuso un gran hogar circular, preparado rebajando el suelo e instalando 
una serie de mampuestos encaminados a mantener la temperatura. En un segundo momento, este hogar dejó de usarse, rellenándose de piedras hasta cota del suelo, perdiendo su función primigenia e integrándose en el piso de la estancia.

La presencia de edificios de diversa complejidad, insertos en un espacio separado por amplias calles a las que se abre algún soportal, responde claramente a un patrón constructivo de origen oriental, habitual en los yacimientos fenicios coetáneos. Por ejemplo, en el yacimiento del Cerro del Villar, en la desembocadura del Guadalhorce -Málaga- (Aubet, 1997), se documentó el mejor y más cercano paralelo de indicios de urbanismo semejante. Allí se localiza una calle de $5 \mathrm{~m}$, que separa edificios complejos de planta ortogonal, que en un caso presenta una estructura simple adosada a uno de los edificios y abierta a la calle. Soportales a base de hiladas de postes han podido ser documentados recientemente en el complejo industrial fenicio de la Pancha, yacimiento recientemente descubierto y ubicado en las inmediaciones de los centros de la desembocadura del río Vélez (Martín, 2005).

\section{Fase III. El uso industrial de la zona.}

Sobre los niveles que amortizaban los edificios y la calle se instalaron una serie de estructuras, aparentemente relacionadas con actividades industriales. Los espacios residenciales quedaron definitivamente en desuso, y en ellos se excavan una serie de estructuras, algunas de ellas de difícil interpretación, con forma de cubetas revestidas de barro, y otras que se pueden interpretar como piroestructuras relacionadas con alguna actividad productiva. A pesar de no haberse conservado material cerámico datante asociado a esta fase, la continuidad estratigráfica existente entre los depósitos, así como la presencia de materiales de época púnica localizados con carácter residual en fosas más tardías, nos permite plantear la continuidad del yacimiento, aunque este aspecto debe ser contrastado en futuras intervenciones.

Del estudio del material cerámico realizado por Ramon se deduce que, salvo casos excepcionales, la cerámica a torno recuperada es de fabricación fenicia. Entre ella se han distinguido individuos procedentes del Mediterráneo central y otros cuyo lugar de producción, seguramente, debe buscarse en Oriente.

En cuanto a las producciones a mano deben destacarse dos hechos. El primero es su abundancia cuantitativa en relación a la cerámica a torno. El segundo, su atribución a producciones artesanales que por su mineralogía cabe ubicar en el propio asentamiento de la Catedral de Ceuta o en su estricta proximidad.

Estos materiales permiten situar un inicio del asentamiento a finales del siglo VIII o inicios del VII a.C., hecho que la cerámica a mano, con ausencias significativas como las retículas bruñidas entre otras, corrobora plenamente.

Este mismo complejo indica que la fase, ya proto-urbana, con calles y estructuras sobre zócalos de piedra rectangulares, pudo implantarse hacia mediados del siglo VII a.C. y que, al menos, en toda su segunda mitad estuvo ocupada.

Otro matiz de interés es el de la procedencia de la cerámica fenicia occidental. Sus características físicas y mineralógicas apuntan claramente a centros de lo que, genéricamente, se ha denominado grupo "Málaga-Granada". Por lo que atañe a vasos fenicios centro-mediterráneos (en especial cartagineses) y orientales su presencia es la normal en los establecimientos occidentales.

Como indicamos al principio, la excavación realizada en la Plaza de la Catedral ha permitido documentar por primera vez en la Ciudad la presencia de niveles del siglo VII a.C. que según los primeros análisis mantienen directas relaciones con las colonias fenicias del litoral malagueño. Se trata pues de un asentamiento de suma importancia en el marco geográfico donde se implanta y donde, junto con un innegable substrato autóctono, la presencia comercial y seguramente también física del factor fenicio, desde sus mismos comienzos y a lo largo de toda la secuencia observada, introducirá aspectos claves de algo, en realidad, mal estudiado: la actividad cultural y económica mixta y las fundaciones de asentamientos conjuntos que obedecieron sobre todo a la conexión citada.

\section{SEPTEM FRATRES}

Las recuperaciones de ánforas destinadas al transporte de las salazones de pescado a mediados del siglo pasado, llevadas a cabo en la dársena del puerto ceutí por Juan Bravo y otros escafandristas del Club de Actividades Subacuáticas (C.A.S), permitió plantear que también se produciría en Ceuta dicho adobo [el garum], producto de las factorías conserveras de entonces, antecesoras de las actuales (Bravo y Muñoz, 1965). No transcurrió mucho tiempo sin que estas hipótesis encontrasen confirmación. Con motivo de la construcción del Parador Nacional "La Muralla" se pudieron observar vestigios de contenedores recubiertos de opus signinum destinados a la elaboración de salazones de pescado. En esta ocasión no sólo se detectaron restos de estas estructuras sino también un buen número de fragmentos anfóricos cuya tipología los relacionaba con claridad con el transporte de salazones. Posac, que dirigió las labores de recuperación y documentación de los hallazgos, recogía en su informe de 1966 cómo "pese a las dificultades derivadas del hecho de emplearse máquinas poderosas para la excavación ha sido posible recoger bastantes restos de cerámica y monedas que permiten seguir una línea cronológica que se inicia en torno a la mitad del siglo I antes de J.C. para llegar hasta finales de los tiempos romanos en los años iniciales del siglo $\mathrm{V}$ de $J C^{\prime \prime}$ (Posac, 1966; 1971). La aparición de estas piletas fue dada a conocer también por otros investigadores (Bravo, 1968). La presencia fundamentalmente de T.S.I. y T.S.G., lucernas de volutas, etc., ha permitido situar la fecha de inicio de la actividad de la factoría en torno al cambio de era (Bernal y Pérez, 1999, 28-29) aunque con las lógicas reservas debido a la falta de contexto estratigráfico de los materiales.

Mientras continuaban produciéndose hallazgos submarinos se produjeron nuevos descubrimientos como consecuencia de los movimientos llevados a cabo en la Plaza de África y la Avda. Alcalde Sánchez Prados para la construcción del aparcamiento subterráneo. Frente al Palacio Municipal apareció un segundo conjunto de piletas del que apenas se conserva una fotografía. A partir de los noventa comienzan una serie de intervenciones arqueológicas en la zona más oriental del Istmo que permiten la documentación de tres nuevos conjuntos de piletas así como de diferentes dependencias asociadas a distintas actividades de la factoría (Hita Ruiz, Villada Paredes, 1994; Bravo et alii, 1995; Villaverde Vega y López Pardo, 1995; Bernal Casasola y Pérez Rivera, 1999).

Desde un punto de vista cronológico Posac había sido sugerido un progresivo desplazamiento hacia el oeste de la presencia romana. Esta hipótesis queda confirmada a partir de esas intervenciones de los noventa en las que se documentó 
que la ocupación de la zona más occidental del Istmo comienza a partir del siglo II d.C. llegando a convertirse en esa centuria y en la siguiente en uno de los centros de producción de salazones más importantes desde el punto de vista regional. Un cese en las actividades productivas a fines del siglo III d.C. y una inmediata recuperación en el siglo IV d.C. han sido propuestos por algunos autores (Bernal Casasola y Pérez Rivera, 1999, 76). El momento final de la producción ha sido situado en distintos momentos del siglo V (Hita Ruiz y Villada Paredes, 1994) o incluso en los inicios del siglo VI d.C. (Bernal Casasola y Pérez Rivera, 1999).

Nuevos restos de esta factoría de salazón han visto la luz recientemente. Efectivamente, en el curso de una visita efectuada por ponentes y asistentes a las I Jornadas de estudio sobre Fortificaciones en junio de 2002 a la zona de las Murallas Reales, ocupadas por el Parador de Turismo "La Muralla", fueron identificados diversos vestigios de la antigua cerca medieval de Ceuta de la que nos ocuparemos un poco más adelante. Diversos lienzos de época califal así como un puerta con arco de herradura enmarcado por un alfiz fueron reconocidos en ese momento (Hita Ruiz y Villada Paredes, 2004 b, 206).

En 2003 fue realizada una intervención que consistió en la limpieza de algunos paramentos, documentación exhaustiva de las estancias (planimetría, fotografía y video) y la realización de cuatro sondeos arqueológicos, tres en la cubierta superior de las Murallas Reales y uno en el umbral de la Puerta, que será el que aquí nos ocupe.

En este sondeo la secuencia documentada comenzaba con la construcción del Parador. Continuaba con varios niveles interpretados como diversas estancias de un acuartelamiento bajo los que se situaban otros de época medieval. En la base de dicha secuencia se documentó una ocupación de época romana que corresponde a la paleoplaya sobre la que se desarrolló la actividad en ese periodo. Habían sido cortados por la cimentación de la muralla califal y en ellos se recuperó una notable muestra de ictiofauna y malacofauna actualmente en proceso de estudio.

Cuatro fases fueron delimitadas en este periodo.

En la primera se identificó una estructura circular, impermeabilizada al interior, a la que llegaba una atarjea construida con tegulae. Esta estructura fue realizada cortando una primera fase de ocupación que fechamos a partir de época flavia. Las ánforas mayoritarias corresponden a tipos Beltrán II A y Dressel 7/12 debiendo destacarse también la presencia de lucernas de volutas y de T.S.G.

Sobre este nivel se sitúa otro (fase II) de similar textura y composición. En él se ha recuperado un conjunto cerámico fechado a partir de la segunda mitad del siglo II d.C. en el que aparecen ya cerámicas africanas de cocina, ausentes en la fase I, algún fragmento de T.S.H. y numerosas cerámicas comunes (imitaciones de cerámicas africanas de cocina, tapaderas Vegas 17, etc.).

Aproximadamente a inicios de la siguiente centuria (fase III) la estructura circular fue abandonada, quedando colmatada con aportes detríticos de variada naturaleza entre los que se han identificado fragmentos de TSC AVC, TSC C, lucernas de disco, cerámicas africanas de cocina y cerámicas comunes. En el interior de la atarjea se localizaron un fragmento de tapadera Vegas 17 y otro de TSH 29/37.

Los niveles más tardíos de época romana (fase IV) han sido alterados por las estructuras medievales, quedando la estructura circular cortada por la muralla califal. La documentación de algunas producciones de T.S.C. D, cerámicas a torno lento y de ánforas tardías testimonia este último momento desaparecido en la secuencia.

Una segunda campaña de excavaciones fue desarrollada en 2005 con objeto de estudiar la secuencia de la zona interior de la Puerta (figura 4). Se llevaron a cabo dos sondeos, A y B. El primero, situado al norte, en las inmediaciones de la Puerta, es la continuación inmediata del sondeo realizado en años precedentes. El sondeo B se ubica hacia el sur, separado del primero por un testigo de metro y medio. Se han documentado diversos periodos de ocupación que, esquemáticamente, son:

Contemporáneo: construcción del Parador de Turismo “La Muralla", Parque de Artillería, enterramientos asociados a las epidemias del siglo XVIII, etc.

Moderno: periodo de construcción y uso del recinto amurallado renacentista.

Medieval: Estructura de la Puerta Califal y sucesivas reestructuraciones (vide infra). En general, los estratos asociados a estos momentos son escasos, destacando algún suelo bien compactado.

Romano. Se diferencian tres fases. La primera se asocia a la construcción de una fosa excavada sobre el sustrato geológico de dos metros de ancho, uno de profundidad y una longitud conservada de unos cuatro metros. Esta obra se mantuvo en uso hasta momentos de mediados del siglo I d.C. en que empezó a usarse como basurero. Sólo contamos pues con un término ante quem para aproximarnos a la fecha de la excavación de esta substrucción.

La segunda fase es precisamente la de uso como basurero de este sector del yacimiento. El material cerámico recuperado permite fechar dicho basurero en la segunda mitad del siglo I d.C.

El tercer momento del primer periodo se asocia a la construcción de un suelo y una canalización. La cronología de que disponemos es el término post quem aportado por los últimos momentos del basurero (inicios del siglo II d.C.). El suelo, de tierra apisonada, y la traída de agua (una serie de tubos cerámicos machihembrados

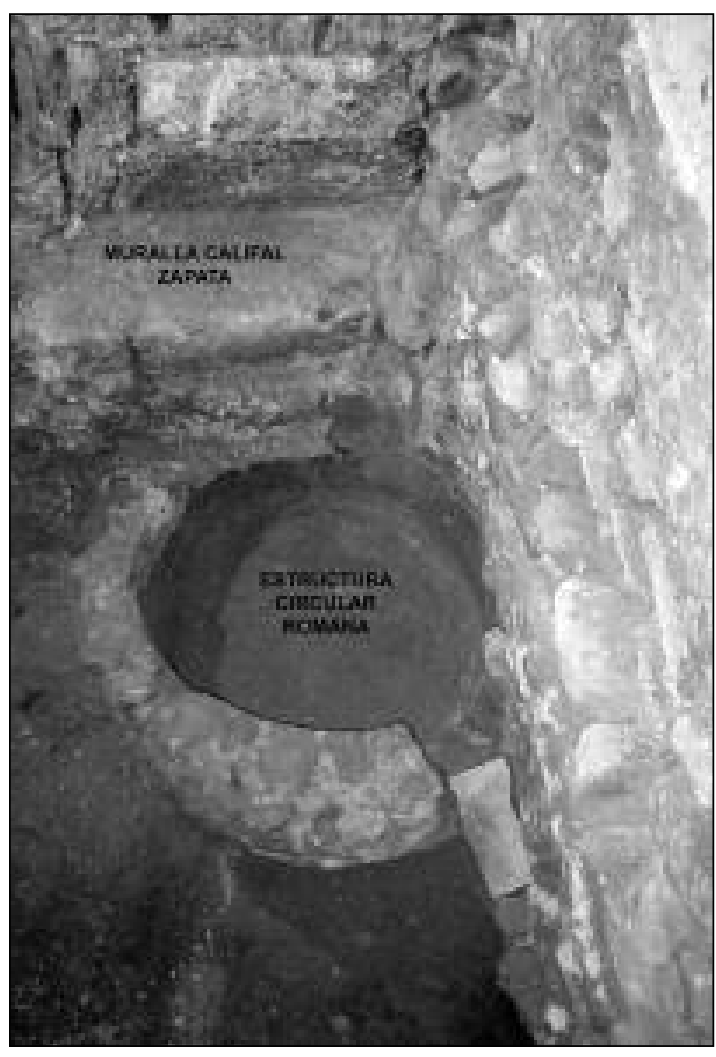

Figura 4. Niveles romanos de la factoría de salazón (excavación arqueológica de 2005). 
cubiertos por ímbrices) pueden ponerse en relación con la pileta circular que se documentó en la excavación precedente. Dicha estructura se colmató en momentos del siglo III d.C., superponiéndose a ella niveles con materiales del siglo IV d.C.

Las dos campañas de excavaciones llevadas a cabo en el Parador de Turismo "La Muralla" han permitido, por primera vez, documentar estratigráficamente la fase altoimperial de Septem de la que únicamente se tenía constancia por las recuperaciones realizadas por Posac.

La ocupación de este espacio parece plenamente atestiguada en época flavia, si bien se han detectado indicios de transformaciones anteriores que se concretan en la realización de infraestructuras relacionadas posiblemente con las necesidades de la factoría. Distintas refacciones y reacondicionamientos tienen lugar, siendo especialmente significativa la construcción de un suelo y de una canalización que, a una cota bastante superior, reproduce sin embargo el trazado y la pendiente de la amortizada.

Hacia el siglo III se aprecian nuevas reestructuraciones en un proceso ya documentado por Bernal y Pérez en las excavaciones del Paseo de las Palmeras. La fase más tardía aparece destruida por la construcción de las fortificaciones medievales, lo que impide aportar datos en torno a la fase bajoimperial de la ocupación.

De otra parte, las excavaciones realizadas aportan información para determinar los límites occidentales de la factoría y el carácter del poblamiento de este sector occidental del Istmo de Ceuta. Así, y aunque no han podido ser documentadas estructuras claramente asociadas a la factoría, el contexto conocido de otros hallazgos (piletas de salazón documentadas por Posac a algunos metros de distancia), la amplia muestra ictiológica y malacológica recuperada, la mayoritaria presencia de ánforas salazoneras y las propias características paleotopográficas del área excavada -una paleoplaya tendida hacia la bahía norte- permiten plantear que la factoría de salazones se extendiese al menos hasta el actual Foso Real e incluso más allá, si tenemos en cuenta las recuperaciones de ánforas en el Angulo de San Pablo.

\section{LA PERLA ENTRE EL PECHO Y LA GARGANTA DEL MUNDO}

La investigación arqueológica sobre este periodo medieval ha tenido gran relevancia tradicionalmente. A los primeros trabajos de Posac siguieron pronto los estudios cerámicos de Fernández Sotelo completados a partir de la década de los 90 por nuevas aportaciones que incidían sobre distintos aspectos (véase bibliografía final).

Cuantitativamente se trata del periodo del que proceden los hallazgos más numerosos, lo que se explica tanto por la propia importancia de la Ciudad medieval como por su propia evolución histórica: conquistada en 1415 por Joao I, el dominio portugués supuso una notable disminución de la población y el abandono de sectores de la ciudad islámica que sólo tardíamente han sido reocupados. Este proceso ha permitido una conservación excepcional de los niveles de ocupación islámicos, especialmente de los más próximos a la conquista.

También los hallazgos medievales son los más numerosos en los últimos años (2000-2005). Así, siguiendo con una tónica habitual en momentos anteriores (Fernández Sotelo, 1988; 2001; 2005), la excavación de diversos silos en la Almina ha permitido la exhumación de una ingente cantidad de objetos muebles. En el caso de las cerámicas, constituyen uno de los más ricos repertorios conocidos en el Mediterráneo Occidental, especialmente en lo que se refiere al último momento de la Ceuta mariní. La mayoría se encuentran aún en proceso de estudio, por lo que no pueden ser objeto de una descripción más precisa hasta el momento.

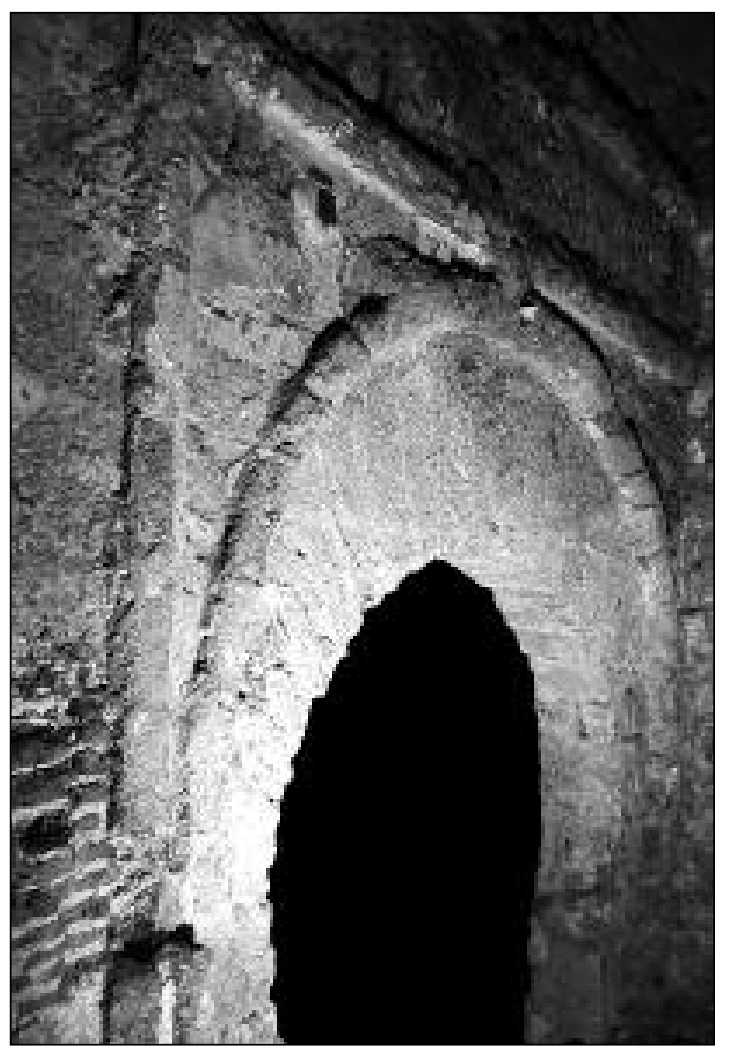

Como hemos indicado, son los momentos más modernos los mejor conocidos (Hita Ruiz y Villada Paredes, 1998; 2004 a; Villada Paredes e Hita Ruiz, 2000 a y 2000 b) lo que contrastaba con la falta de registro material que pudiera ser datado antes de la conquista de los cordobeses (391 H./931). En 2004, una excavación llevada a cabo en la Almina permitió recuperar en una fosa circular de escasas dimensiones, utilizada como "vertedero" en el último momento, un significativo conjunto de piezas que han podido ser fechadas en momentos anteriores a esta conquista. A pesar del escaso repertorio conservado, su interés es evidente por confirmar datos recogidos por los cronistas que señalan la influencia andalusí en momentos previos a la conquista y que parece reconocerse también en el registro material estudiado (Hita Ruiz, Suárez Padilla y Villada Paredes, en prensa).

Tras la conquista de los omeyas cordobeses se construye por orden de al Nasir una muralla de piedra culminada por su hijo al Hakam II. Punto de vital importancia para la política norteafricana omeya, el esfuerzo realizado por los omeyas para construir esta cerca es resaltado en diversas fuentes.

De esta muralla se conocían diversos tramos (figura 5). En la esquina noroccidental del recinto se identificaron una torre y un lienzo. El tramo occidental de dicha torre quedaba oculto por el adosamiento de una nueva fábrica que formaba parte del Baluarte de los Mallorquines, fortificación del Conjunto Monumental de las Murallas Reales. Estos elementos pronto quedaron ocultos tras la construcción del Club de Actividades Subacuáticas de Ceuta (Terrase, 1962) y sólo en 2003 han sido puestos de nuevo al descubierto tras el derribo de esta construcción adosada.

El lienzo que discurre hacia oriente conecta con otra torre, apenas visible entonces, de alrededor de $3 \mathrm{~m}$ de frente a la que se adosó una nueva construcción a fin de reforzarla. La distancia entre 
torres es de 21 m aproximadamente (Hita y Villada, 2004c). En 2004 fue excavado por J. Suárez este sector. Además de distintas estructuras más modernas fueron exhumados los restos de la torre antes indicada que había sufrido diversas remodelaciones más tardías.

También en el frente norte se localizó en el interior del Baluarte de los Mallorquines en 2002 un nuevo fragmento de lienzo con características similares, y por tanto idéntica cronología a los descritos por Terrasse. Si esta datación es correcta pertenecerían al alcázar califal. Tras la construcción del Baluarte fue integrado en éste, siguiendo un proceso similar al que describiremos en el sector oeste.

Un nuevo tramo en el sector oriental de este recinto fue descubierto hace algunos años por Fernández Sotelo, si bien fue datado por él como perteneciente a la fortificación romana. En este caso se trata de un lienzo de más de 45 metros con dos torres. El frente de las mismas es de aproximadamente 2,90 m. La torre sur sufrió numerosas reformas que se evidencian por la presencia en su forro de sillares de distinta naturaleza y dimensiones a los de la cerca original. La cerca sigue la pendiente natural del terreno y, a medida que nos aproximamos al sur, se encuentra peor conservada.

Pero los hallazgos más espectaculares corresponden al sector occidental de la muralla. Como señalamos antes, fueron identificados dos nuevos tramos de esta cerca que acabaron por integrarse en el conjunto de murallas manuelinas que defienden la Ciudad por el llamado Frente de Tierra.

El primero de ellos, en el interior del Baluarte de la Coraza, corresponde a un tramo de cortina que en la zona superior se remata con tres cordones horizontales. En este punto el espesor de la muralla califal es de aproximadamente un metro y medio, y conserva un alzado aproximado de unos nueve metros.

El otro tramo identificado se encuentra situado en la zona central del flanco occidental. Corresponde a un lienzo con una altura conservada de al menos siete metros y trece de recorrido visible, en el que se abre una de las puertas de acceso a la ciudad formada por un arco de herradura enmarcado por un alfiz.

La puerta se encuentra flanqueada por la misma cortina que justo a su lado derecho realiza un quiebro, adelantándose respecto al lienzo de muralla que discurría hasta la mencionada puerta. Las dovelas presentan aún restos de pintura roja que denotan su primitiva decoración. Sobre la puerta de acceso exterior se conserva el hueco en el que debió ir situada la lápida fundacional, hoy perdida.

A través de esta puerta se ingresa a un primer espacio cubierto por bóveda de arista rebajada.

Las dos excavaciones realizadas en este lugar han permitido poner al descubierto una de las puertas de acceso a la medina, construida en época califal pero con múltiples modificaciones posteriores ya en época medieval, y conocer con mayor profundidad el proceso constructivo de las fortificaciones renacentistas.

En su fase final el acceso desde la Puerta al interior de la medina se realiza a través de un doble recodo. En nuestra opinión, en época califal debió ser de ingreso directo o de recodo simple, correspondiendo la adición del siguiente tramo a momentos posteriores. La preservación de este flanco occidental, en un excelente estado de conservación, ha sido posible al construirse la Muralla Real lusitana adelantada respecto a la cerca califal, incorporándose esta última al nuevo dispositivo defensivo al rellenarse el hueco entre ambas con tierras, piedras y restos de los elementos demolidos de la zona superior.

Todos estos datos permiten restituir el trazado de la cerca omeya. El recinto tenía una planta rectangular de 350 por 200 metros aproximadamente. En su ángulo noroccidental se encontraba el alcázar. La medina ceutí delimitada por esta cerca tendría una extensión de aproximadamente siete hectáreas (similar a la de ciudades como Gibraltar, Elvas, Niebla, etc.). En ella se sitúan los edificios públicos más representativos del poder político (el alcázar) y religioso (la mezquita aljama), así como algunas de las residencias de los notables de la ciudad.

La muralla está construida con sillares de calcarenita, en algunos casos calzados con ripios, de procedencia alóctona, que forran un mampuesto interior realizado con piedras sin trabajar. Las dimensiones de los sillares es bastante homogénea, con una longitud entre 92 y $99 \mathrm{~cm}$ en general, una altura en torno a los $30 \mathrm{~cm}$ y un grosor de aproximadamente de $20 \mathrm{~cm}$, aunque algunos son mayores. La técnica edilicia se basa en el empleo de un aparejo a soga y doble o triple tizón. Esta disposición se da únicamente en las zonas visibles. Los lienzos se refuerzan con torres cuadrangulares que, aunque en los diferentes sectores del recinto tienen dimensiones distintas, poseen una cierta regularidad. Si estableciéramos una media podríamos decir que se disponen aproximadamente cada $20 \mathrm{~m}$ en los lienzos, que su frente ronda en torno a los $3 \mathrm{~m}$ y que su adelantamiento respecto a la cortina es de en torno a 1,70 m.

Promovidas por el estado omeya, todas estas construcciones guardan entre sí estrechos vínculos ya que, a su misión defensiva, unen un marcado carácter simbólico. Así, ni la elección del material empleado, ni el aparejo constructivo típicamente cordobés, ni la tipología de las planimetrías utilizadas, son fruto del azar. Al margen de su carácter defensivo, estas fortalezas son la concreción material del dominio político omeya en la zona del Estrecho y ponen de manifiesto su capacidad técnica y económica y su poderío militar.

Otro aspecto de interés del urbanismo ceutí es el estudio de las necrópolis. Han sido estudiadas fundamentalmente a partir de la descripción de al Ansari.

Durante este último lustro dos excavaciones (Real 41 y Fructuoso Miaja) han permitido conocerlas con mayor amplitud. El periodo de uso de ambas comienza poco después del periodo califal y se extiende hasta los últimos momentos de la Ceuta islámica posiblemente. Se trata de un conjunto de inhumaciones realizadas en fosa simple, a menudo delimitadas por piedras de mediano tamaño o por tejas, sin ajuar, en las que el cadáver se depositaba directamente en el suelo, quizás con unas parihuelas como denotan la presencia de pequeños clavos en el interior de las mismas. Los cuerpos aparecen en posición lateral con los rostros vueltos hacia el este, los brazos extendidos a lo largo del cuerpo y las manos sobre la pelvis. No obstante, han sido detectadas ciertas remociones de los cadáveres relacionadas en buena medida con el intenso uso de los cementerios. Una de las características más sobresalientes en el ritual de enterramiento consiste en la aportación intencionada de fragmentos de conchas. Esta costumbre cuenta con paralelos en necrópolis algecireñas de época mariní.

Los estudios efectuados, aún en fase de publicación, han permitido obtener un importante caudal de datos en torno a las características antropológicas, patologías y dieta de los individuos enterrados.

Aunque los cementerios ceutíes habían podido ser estudiados a través de distintas crónicas y a través de la excavación de algunos de ellos en el Monte Hacho, desgraciadamente en bastante mal estado de conservación, los nuevos datos 
obtenidos aportan una valiosa información para conocer la evolución del urbanismo ceutí, el ritual de enterramiento y sus conexiones con localidades próximas así como las características físicas de la población que habitó Ceuta durante ese periodo.

\section{CONCLUSIONES}

Como hemos expuesto a través de las páginas precedentes, el caudal de información aportado por la investigación arqueológica en los últimos años ha sido muy relevante. Pero estos datos, por significativos que puedan parecer, cobran únicamente sentido cuando son articulados en el marco general de un estudio sobre la historia de la Ciudad. Sólo desde esa comprensión global de nuestra historia urbana es posible detectar lagunas de conocimiento, establecer hipótesis de trabajo e identificar cuáles serían los procedimientos para verificarlas, etc. En definitiva, no parece razonable conformarnos con una mera "documentación de los solares" desarticulada, ininteligible por la disparidad de intereses y sistemas de registro usados y, la mayor parte de las ocasiones, tan volcada en la microhistoria que sus resultados rayan a veces en lo meramente anecdótico. La rígida separación a veces planteada entre la llamada "arqueología de gestión" y "arqueología de investigación" no es justificable desde nuestro punto de vista. Así, si bien las excavaciones, principalmente de "urgencia", se han multiplicado en los últimos años con la consiguiente "destrucción controlada" del patrimonio arqueológico, nuestro conocimiento histórico no ha experimentado un avance proporcional. La presentación efectuada, a pesar de su brevedad, revela con claridad el potencial que la investigación desarrollada por la arqueología urbana en Ceuta tiene para la comprensión de nuestra historia y la necesidad de continuar con esta línea de trabajo en el futuro.

En cualquier caso si la gestión arqueológica es inconcebible sin un proyecto de investigación que le dé sentido, es evidente que otros aspectos también son necesarios. Así, la arqueología urbana en Ceuta no se limita solamente a esta faceta de la investigación sino que ha abordado, también en este periodo, actividades como la puesta en valor de diversos yacimientos (yacimiento protohistórico de la Plaza de la Catedral, Museo de la Basílica Paleocristiana, Baños árabes, etc.) y otras actividades de difusión de este patrimonio (visitas a yacimientos en proceso de excavación, publicaciones tanto científicas como divulgativas, organización de jornadas, congresos, conferencias, etc., actividades para escolares, etc.). Su análisis, sin embargo, excede los límites de este artículo y será objeto de próximos trabajos.

\section{BIBLIOGRAFÍA}

AA.VV., (1998): Homenaje al profesor Carlos Posac Mon, Ceuta.

ALCALÁ VELASCO, L. L. (1998): "La protección del patrimonio arqueológico ceutí: Una ordenanza vigente y una asignatura pendiente", en AA.VV., Homenaje al profesor Carlos Posac Mon, Ceuta, vol. III, pp. 359-367.

ARÓstegul, A. (1998): "La obra de Posac en Ceuta y por Ceuta", en AA.VV., Homenaje al profesor Carlos Posac Mon, Ceuta, vol. I, pp. 31 38.

AUBET, M. E. (1997): "Un lugar de mercado en el Cerro del Villar", en Aubet, M. E. (coord.) Los fenicios en Málaga, Málaga, pp. 197-213.

BERNAL CASASOLA, D. (1994): "Marcas sobre materiales de construcción en Ceuta y la problemática de la necrópolis de las Puertas del Campo", Transfretana 6, pp. 61-80.

BERNAL CASASOLA, D. (1997): "Las ánforas romanas bajoimperiales y tardorromanas del Museo Municipal de Ceuta", en Ánforas del Museo de Ceuta, Ceuta, pp. 61-129.

BERNAL CASASOLA, D. (2000): "Hallazgos arqueológicos y estado de la cuestión sobre la presencia de fenicio-púnicos en Ceuta", en Actas del IV Congreso Internacional de Estudios Fenicios y Púnicos, Cádiz, pp. 1.137-1.152.

BERNAL CASASOLA, D. (2004): Las cartas arqueológicas en España. Recientes experiencias en Andalucia y Ceuta, «AAAd», 58, pp. 83-110.

BERNAL CASASOLA, D. (ed., 2004): Juan Bravo y la arqueología subacuática en Ceuta. Un homenaje a la perseverancia, Ceuta.

BERNAL CASASOLA, D., HOYO CALLEJA, J. DEL y PÉREZ RIVERA, J.M. (1998): “Isis en Mauretania Tingitana: un nuevo testimonio epigrafico de su culto procedente de Septem Fratres (Ceuta)" , L'Africa romana, Atti del XII convegno di studio, Olbia, 12-15 dicembre 1996, Sassari, pp. 1139-1162.

BERNAL CASASOLA, D. y PÉREZ RIVERA, J.M. (1998): "Aportaciones al poblamiento de época medieval en Ceuta: Un nuevo yacimiento arqueológico en las proximidades de La Tortuga", en AA.VV., Homenaje al profesor Carlos Posac Mon, Ceuta, vol. I, pp. 429-438.

BERNAL CASASOLA, D. y PÉREZ RIVERA, J.M. (1999): Un viaje diacrónico por la historia de Ceuta. Resultados de las intervenciones arqueológicas en el Paseo de las Palmeras, Ceuta.

BRAVO PÉREZ, J. (1968) : "Fábrica de salazones en la Ceuta romana", CRIS, Revista del Mar, p. 40.

BRAVO PÉREZ, J. y MUÑOZ, R., (1965): Arqueología submarina en Ceuta, Madrid.

BRAVO PÉREZ, J. et alii (1995): "Nuevos datos sobre la economía del territorio ceutí en época romana: las factorías de salazón", Actas del ॥l Congreso Internacional "El Estrecho de Gibraltar", Tomo I, Ceuta.

FERNÁNDEZ SOTELO, E. A. (1988): Ceuta medieval: aportación al estudio de las cerámicas, S. X-XV, Ceuta.

FERNÁNDEZ SOTELO, E. A. (1991): La Basílica. tardorromana de Ceuta, Ceuta.

FERNÁNDEZ SOTELO, E. A. (1994): Un vertedero del siglo III en Ceuta, Ceuta.

FERNÁNDEZ SOTELO, E. A. (1995): "La basílica tardorromana de Ceuta", en I/ Congreso Internacional «El Estrecho de Gibraltar» (Ceuta, Noviembre 1990), Madrid, pp. 509-526.

FERNÁNDEZ SOTELO, E. A. (1998): "Cerámica hispano-musulmana: Algo más sobre la cerámica bícroma", en AA.VV., Homenaje al profesor Carlos Posac Mon, Ceuta, pp. 349-358.

FERNÁNDEZ SOTELO, E. A. (2000): Basílica y necrópolis paleocristianas de Ceuta, Ceuta. 
FERNÁNDEZ SOTELO, E.A. (2001): Los silos en la arqueología ceutí (I), Ceuta.

FERNÁNDEZ SOTELO, E.A. (2005): Los silos en la arqueología ceutí (II), Ceuta.

FERNÁNDEZ SOTELO, E.A. y ROVIRA LLORENS, S. (1997): Las arracadas de Ceuta, Ceuta.

GOZALBES CRAVIOTO, C. (1995): El urbanismo religioso y cultural de Ceuta en la Edad Media, Ceuta.

GOZALBES CRAVIOTO, C. (1998): "El gran aljibe medieval de la Almina de Ceuta", en AA.VV., Homenaje al profesor Carlos Posac Mon, Ceuta, vol. I, pp. 471-484.

GOZALBES CRAVIOTO, E. (1990): El nombre romano de Ceuta, de Septem Fratres a Ceuta, 1990.

GOZALBES CRAVIOTO, E., (2000): Economía de la Mauritania Tingitana (siglos I a. de C.-Il d. de C.), Ceuta.

HITA RUIZ, J. M. y VILLADA PAREDES, F. (1994): Excavaciones arqueológicos en el istmo de Ceuta, Ceuta.

HITA RUIZ, J. M. y VILLADA PAREDES, F. (1998): "Cerámica con cubierta estannífera de Huerta Rufino (Ceuta)", en AA.VV., Homenaje al profesor Carlos Posac Mon, Ceuta, vol. I, pp. 443-470.

HITA RUIZ, J. M. y VILLADA PAREDES, F., (2000): Un aspecto de la sociedad ceutí en el siglo XIV: los espacios domésticos, Ceuta.

HITA RUIZ, J. M. y VILLADA PAREDES, F., (2004a): "Entre el Islam y la Cristiandad. Cerámicas del siglo XV en Ceuta: Avance preliminar", en AA.VV. Cerámicas islámicas y cristianas a finales de la Edad Media. Ceuta, 13-16 de noviembre, 2002, Ceuta.

HITA RUIZ, J. M. y VILLADA PAREDES, F., (2004b): "Informe sobre la intervención arqueológica en el Parador de Turismo "La Muralla" de Ceuta", en Actas de las I Jornadas de estudios sobre fortificaciones y memoria arqueológica del hallazgo de la muralla y Puerta Califal de Ceuta, Ceuta, pp. 205-243.

HITA RUIZ, J. M. y VILLADA PAREDES, F., (2004c): "En torno a las murallas de Ceuta. Reflexiones sobre el amurallamiento de Septem Fratres y la cerca omeya de Sabta", en Actas de las I Jornadas de estudios sobre fortificaciones y memoria arqueológica del hallazgo de la muralla y Puerta Califal de Ceuta, Ceuta, pp. 17-52.

HITA RUIZ, J. M., SUÁREZ PADILLA, J y VILLADA PAREDES, F. (en prensa): "La perla entre el pecho y la garganta del mundo: Una relectura de la historia de Ceuta desde la conquista árabe hasta la fitna a raíz de los datos arqueológicos", Cuadernos de Madinat al-Zahra, en prensa.

HOYO CALLEJA, J. DEL, BERNAL CASASOLA, D. y PÉREZ RIVERA, J. M. (1996): "Tres inscripciones latinas inéditas procedentes de la basílica tardorromana de Ceuta (Mauretania Tingitana)", Melanges de la Casa de Velázquez, pags. 71-84.

MARTín, E. (2005): "Nuevos yacimientos fenicios en el contexto de la costa de Vélez-Málaga (Málaga)", en AA.VV., XVII Cursos de verano de la Universidad de Granada en Ceuta, Ceuta, pp. 101-105.

MARTínEZ MAGANTO, J. y GARCÍA GIMÉNEZ, R. (1997): "El conjunto de ánforas altoimperiales de salazón de Ceuta", Ánforas del Museo de Ceuta, Ceuta, pp. 7-60.

PARDO NARANJO, A. I., GARCíA JIMÉNEZ y MARTíNEZ MAGANTO, J. (1998): "Alteraciones y problemas de conservación del material anfórico romano del Museo de Ceuta", en AA.VV., Homenaje al profesor Carlos Posac Mon, Ceuta, vol. III, pp. 369-380.

PÉREZ RIVERA, J. M., L. y BERNAL CASASOLA, D. (1998): “La factoría de salazones romana de Septem Fratres: Novedades de las excavaciones arqueológicas en el Paseo de las Palmeras, núms. 16-24", en AA.VV., Homenaje al profesor Carlos Posac Mon, Ceuta, vol. I, pp. $249-264$.

PÉREZ RIVERA, J. M., HOYO CALLEJA J. DEL y BERNAL CASASOLA, D. (1999): “Epígrafe inédito hallado en Ceuta. Acerca del estatuto jurídicoadministrativo de Septem Fratres", Latomus. Revue d'Études Latines.

POSAC MON, C. (1960): "Datos para la arqueología musulmana de Ceuta", en Hesperis-Tamuda, I, 1960, pp. 157-164.

POSAC MON, C. (1962): Estudio arqueológico de Ceuta, Ceuta.

POSAC MON, C. (1966): Informe arqueológico año 1966, (inédito).

POSAC MON, C. (1971): "La arqueología en Ceuta entre 1960-1970", Noticiario Arqueológico Hispánico, 15, pp. $229-235$.

QUEROL, M. A. y MARTíNEZ DÍAZ, B. (1996): La gestión del Patrimonio Arqueológico en España, Madrid.

RAMON TORRES, J. (2004): "Las ánforas fenicio-púnicas de Ceuta", en D. Bernal (ed.), Juan Bravo y la arqueología subacuática en Ceuta, Ceuta: Instituto de Estudios Ceutíes, pp. 95-106.

RODRÍGUEZ TEMIÑO, I. (2003): Arqueología urbana en España, Madrid.

ROSELLÓ, E. (1992): "La ictiofauna recuperada en el Istmo de Ceuta", Arqueofauna, 1, pp. 23-32.

ROSELLÓ BORDOY, G., (1998): "Más sobre el brocal de pozo almohade de Ceuta", en AA.VV., Homenaje al profesor Carlos Posac Mon, Ceuta, vol. I, pp. 359-368.

TERRASSE, H. (1962) : "Un vestige de fortifications oméyaides de Ceuta", en al Andalus, XXVII, pp. 244-246.

VILLADA PAREDES, F. e HITA RUIZ, J.M. (1994): "El asentamiento romano de Ceuta", en Mastino, A. y Ruggeri, P. (eds.), L'Africa romana: Atti del X Convengo di Studio, Oristano, 11-13 dicembre 1992, Sassari, pp. 1207-1240.

VILLADA PAREDES, F. e HITA RUIZ, J.M. (2000a): "Una aproximación al estudio de la cerámica en la Ceuta mariní", en AA.VV., Cerámica nazari y marini, Ceuta, pp. 291-328.

VILLADA PAREDES, F. e HITA RUIZ, J.M. (2000b): "Ceuta durante el periodo mariní: estado de la cuestión y nuevas perspectivas", en Lorenzo Cara Barrionuevo (coord.), Ciudad y territorio en al Andalus, pp. 218-244.

VILLADA PAREDES, SUÁREZ PADILLA, J. y BRAVO JIMÉNEZ, S. (en prensa): "Nuevos datos sobre las factorías de salazones de Septem Fratres", en Lagóstena, L., Bernal, D. y Arévalo, A., Cetariae. Salsas y salazones de pescado en occidente en la Antigüedad (Cádiz, 7-9 noviembre dé 2005). 
VILLADA PAREDES, F., RAMON TORRES, J. y SUÁREZ PADILLA, J. (en prensa): "Un nuevo asentamiento del siglo VII a.C. en Ceuta", VI Congreso Internacional de Estudios Fenicios y Púnicos (Lisboa, 2005).

VILLAVERDE VEGA, N. y LÓPEZ PARDO, F. (1995): "Una nueva factoría de salazones en Septem Fratres. El origen de la localidad y la problemática de la industria de salazones en el Estrecho durante el Bajo Imperio", "I Congreso Internacional El Estrecho de Gibraltar, pp. 455-472.

VILLAVERDE VEGA, N. (2001): Tingitana en la Antigüedad Tardía (siglos III-VII). Auctoctonía y Romanidad en el Extremo Occidente Mediterráneo, Madrid. 